\title{
PERFIL DOS PACIENTES ADULTOS COM ACIDENTE VASCULAR ENCEFÁLICO TRATADOS EM UMA CLÍNICA-ESCOLA DE FISIOTERAPIA
}

\author{
PROFILE OF ADULT PATIENTS WITH STROKE TREATED IN A CLINICAL SCHOOL OF \\ PHYSICAL THERAPY
}

\author{
Rafael Alves Barbosa $\mathrm{a}^{\mathrm{a}^{*}}$, Thiago Brasileiro Vasconcelos ${ }^{\mathrm{b}^{* *}}$, Cristiano Teles de Sousa $^{\mathrm{c}^{*}}$, Micheline \\ Freire Alencar Costa ${ }^{\mathrm{d}^{*}}$, Mayara Paz Albino dos Santos ${ }^{\mathrm{e}^{* *}}$, Vasco Pinheiro Diógenes Bastos ${ }^{\mathrm{f}^{*}}$

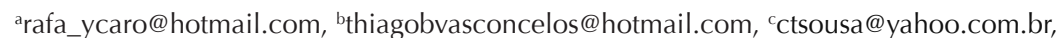 \\ dmichelinefreire@gmail.com, emayara_paz@yahoo.com.br, ${ }^{\mathrm{f}}$ vascodiogenes@yahoo.com.br \\ *Centro Universitário Estácio do Ceará - Fortaleza (CE), Brasil \\ **Universidade Federal do Ceará - Fortaleza (CE), Brasil
}

Data de recebimento do artigo: 17/08/2015

Data de aceite do artigo: 19/11/2015

\section{RESUMO}

Este estudo teve como objetivo traçar um perfil epidemiológico de pacientes com sequelas do acidente vascular encefálico (AVE) tratados em uma clínica-escola de fisioterapia. Trata-se de um estudo de caráter documental e transversal, com estratégia de análise quantitativa dos dados. A média de idade foi de $53,77( \pm 9,52)$ anos, com predomínio do acidente vascular encefálico isquêmico (AVEi) $(69,23 \%$; n=9); a profissáo de comerciante associada a pessoas do sexo masculino apresentou maior incidência (23,08\%; $\mathrm{n}=3)$; o fator de risco mais expressivo foi a hipertensão arterial sistêmica $(61,54 \% ; \mathrm{n}=8)$; das sequelas, marcha helicoidal $(46,15 \% ; n=6)$; hemiplegia desproporcional incompleta à esquerda $(23,08 \% ; n=3)$ e à direita $(23,08 \% ; n=3)$, e como tipo de tratamento, a cinesioterapia $(69,23 \% ; n=9)$. Foi constatado como perfil epidemiológico pacientes do sexo masculino com idade média de 53,77 anos, diagnosticados com AVCi e tendo como profissão o comércio. Entre as sequelas relacionadas, destacou-se a hemiplegia desproporcional incompleta à esquerda e à direita e marcha helicoidal.

Palavras-chave: Acidente vascular cerebral; fisioterapia; hemiplegia.

\section{ABSTRACT}

This study aimed to delineate the specific epidemiological profile of patients with sequelae of stroke treated in a clinical school of physical therapy. This is a documentary study with a cross-sectional quantitative analysis of the results. The average age of the patients was $53.77( \pm 9.52)$ years, with prevalence of ischemic stroke $(69.23 \% ; n=9)$; the profession of merchant, associated with males with ischemic stroke, had the higher incidence $(23.08 \% ; \mathrm{n}=3)$. The most significant risk factor was the hypertension $(61.54 \% ; \mathrm{n}=8)$; and the most significant sequelae were hemiplegic gait $(46.15 \% ; n=6)$ and unequal motor movement on the left $(23.08 \% ; \mathrm{n}=3)$ and right $(23.08 \%, \mathrm{n}=3)$ side of the body; and kinesiotherapy was the predominant type of treatment $(69.23 \%, n=9)$. Male patients with a mean age of 53.77 years, merchants, diagnosed with ischemic stroke were the epidemiological profile of this research. Between the related sequelae, the unequal motor movement on the left and right side of the body and the hemiplegic gait were enhanced.

Keywords: Stroke; physical therapy; hemiplegia. 


\section{Introdução}

O acidente vascular encefálico (AVE) descreve um comprometimento neurofuncional, em que ocorre uma interrupçáo do fluxo sanguíneo na regiáo encefálica por duas formas: sendo anóxico-isquêmicas - resultado da falência de fluxo sanguíneo para suprir o tecido cerebral com oxigênio e substratos - ou hemorrágicas - devido a um rompimento vascular ${ }^{1,2}$.

Das doenças crônico-degenerativas, as cerebrovasculares constituem a terceira causa de morte no mundo, precedida pelas cardiopatias e o câncer. O AVE isquêmico (AVEi), ou hemorrágico, transitório ou definitivo, configura-se como a doença cerebrovascular que apresenta maior incidência e morbidade, resultando em incapacidades ${ }^{3}$.

Castro, Epstein e Sabino ${ }^{4}$ classificam os fatores de risco para o AVE em "não modificáveis, modificáveis e outros". Como riscos não modificáveis, tem-se a idade, sexo, raça, etnia e hereditariedade, nos quais a raça negra e o sexo masculino apresentam maior incidência. Já como riscos modificáveis, tem-se como principal a hipertensão arterial, que aumenta três vezes o risco de acometimento.

Polese $e^{5}$ acrescenta que a arteriosclerose, hipertensão arterial sistêmica (HAS), diabetes mellitus (DM), tabagismo, hipercolesterolemia, obesidade, valvulopatias cardíacas, hereditariedade e sedentarismo são os principais fatores de risco para o AVE. Cancela ${ }^{6}$ confirma tal informação e acrescenta os seguintes fatores: etilismo, cocaína arritmias cardíacas, anticoncepcionais orais e fator idade.

Sendo assim, o presente estudo teve como objetivo analisar o perfil dos pacientes adultos com AVE tratados em uma clínica-escola de fisioterapia.

\section{Metodologia}

Estudo de caráter documental e transversal com abordagem quantitativa, realizado no período de agosto a outubro de 2010, em consonância com a aprovaçáo do projeto pelo Comitê de Ética em Pesquisa do Centro Universitário Estácio do Ceará (Protocolo no 055/10).

A população foi composta pelos pacientes adultos, portadores de sequelas por AVE, que fizeram tratamento na clínica-escola de fisioterapia do Centro
Universitário Estácio do Ceará (Fisiofic), situada na cidade de Fortaleza/CE, durante o período de janeiro a novembro de 2009.

Foram incluídos na amostra os prontuários dos pacientes com AVE cujas idades estavam situadas entre 18 e 65 anos, independente da escolaridade, sexo, estado civil ou renda familiar, e foram excluídos os que estavam fora dessa faixa etária, além dos que estavam com o preenchimento incompleto.

Os dados foram coletados através de um formulário, sendo as informaçôes coletadas a partir da listagem dos prontuários que estavam em conformidade com os critérios de inclusão. Foram utilizadas as seguintes variáveis: tipos de AVE, incidência quanto à idade, sexo, período de tratamento e tipo de tratamento empregado.

\section{Resultados}

A amostra pesquisada apresentou uma média de idade de 53,77 $( \pm 9,52)$ anos. O sexo masculino foi predominante, com 53,84\% ( $\mathrm{n}=7$ ) dos indivíduos (Gráfico 1).

Gráfico 1: Distribuição da amostra segundo o sexo e o tipo de AVE.

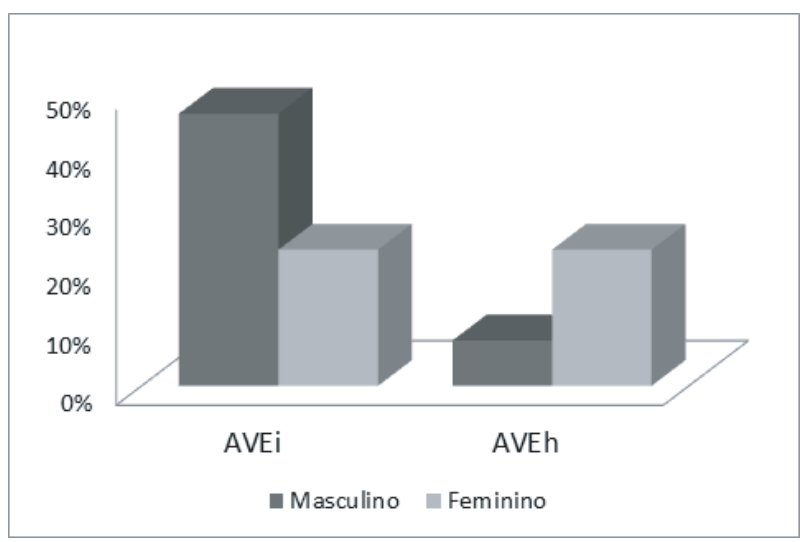

AVEh: acidente vascular encefálico do tipo hemorrágico (coluna cinza-claro); AVEi: acidente vascular encefálico do tipo isquêmico (coluna cinza-escuro).

Ao relacionar os dados - profissáo, sexo e tipo de AVE -, foi possível verificar que a profissão de comerciante associada a pessoas do sexo masculino com AVEi foi a que apresentou maior incidência, com 30,77\% $(n=4)$, sendo essa associação a de maior prevalência no estudo (Tabela 1).

Tabela 1: Distribuição de dados segundo a profissão, sexo e tipo de AVC.

\begin{tabular}{lcccccc} 
& \multicolumn{2}{c}{ AVEi } & \multicolumn{2}{c}{ AVEh } & \multicolumn{2}{c}{ Total } \\
Profissáo & Masc. & Fem. & Masc. & Fem. & Fa & F\% \\
Comerciante & $23,08 \%$ & --- & --- & $7,69 \%$ & 4 & $30,77 \%$ \\
Técnico de enfermagem & --- & $7,69 \%$ & --- & --- & 1 & $7,69 \%$ \\
Mecânico de máquinas pesadas & --- & --- & $7,69 \%$ & --- & 1 & $7,69 \%$ \\
Professor & $7,69 \%$ & $7,69 \%$ & --- & $7,69 \%$ & 3 & $23,08 \%$
\end{tabular}

continua... 
Tabela 1: Continuação.

\begin{tabular}{lcccccc} 
& \multicolumn{2}{c}{ AVEi } & \multicolumn{2}{c}{ AVEh } & \multicolumn{2}{c}{ Total } \\
Sociólogo & $7,69 \%$ & --- & -- & -- & 1 & $7,69 \%$ \\
Médico & --- & --- & --- & $7,69 \%$ & 1 & $7,69 \%$ \\
Motorista de ônibus & $7,69 \%$ & --- & --- & --- & 1 & $7,69 \%$ \\
Costureiro & --- & $7,69 \%$ & --- & --- & 1 & $7,69 \%$ \\
Total & $46,16 \%$ & $23,08 \%$ & $7,69 \%$ & $23,08 \%$ & 13 & $100 \%$ \\
\hline
\end{tabular}

AVEi: acidente vascular encefálico do tipo isquêmico; AVEh: acidente vascular encefálico do tipo hemorrágico; Masc.: masculino; Fem.: feminino; Fa: frequência absoluta; F\%: frequência relativa.

Com relação à história da doença familiar, foi possível verificar que 38,46\% $(\mathrm{n}=5)$ da amostra fazia referência à HAS, seguido da DM, 30,77\% ( $n=4)$ e do AVE, com 15,38\% $(\mathrm{n}=4)$. Em relaçấo às doenças pré-existentes, foi detectado que a HAS apresentou novamente a maior prevalência $(61,54 \%$; $\mathrm{n}=8)$, seguida pela DM e osteoporose $(15,38 \%$; $=2)$.
Segundo a topografia do AVE, foi possível evidenciar que a hemiplegia desproporcional incompleta à esquerda $(23,08 \% ; n=3)$ e à direita $(23,08 \% ; n=3)$ foram as de maior prevalência no estudo, sendo que no AVEi essa hemiplegia foi a mais encontrada $(69,23 \% ; n=9)$ (Tabela 2).

Tabela 2: Distribuição de dados segundo a topografia.

\begin{tabular}{|c|c|c|c|c|c|c|}
\hline \multirow[b]{2}{*}{ Topografia } & \multicolumn{2}{|c|}{ AVEi } & \multicolumn{2}{|c|}{ AVEh } & \multicolumn{2}{|c|}{ Total } \\
\hline & $\mathrm{Fa}$ & F \% & $\mathbf{F a}$ & F \% & $\mathrm{Fa}$ & F \% \\
\hline $\begin{array}{l}\text { Hemiparesia desproporcional } \\
\text { incompleta à esquerda }\end{array}$ & 1 & $7,69 \%$ & --- & --- & 1 & $7,69 \%$ \\
\hline $\begin{array}{l}\text { Hemiparesia desproporcional } \\
\text { completa à esquerda }\end{array}$ & 1 & $7,69 \%$ & -- & --- & 1 & $7,69 \%$ \\
\hline $\begin{array}{l}\text { Hemiparesia desproporcional } \\
\text { incompleta à direita }\end{array}$ & -- & -- & 1 & 7,69\% & 1 & $7,69 \%$ \\
\hline $\begin{array}{l}\text { Hemiplegia proporcional } \\
\text { completa à esquerda }\end{array}$ & -- & --- & 1 & 7,69\% & 1 & $7,69 \%$ \\
\hline $\begin{array}{l}\text { Hemiplegia proporcional } \\
\text { incompleta à esquerda }\end{array}$ & 2 & $15,38 \%$ & -- & --- & 2 & $15,38 \%$ \\
\hline $\begin{array}{l}\text { Hemiplegia desproporcional } \\
\text { completa à esquerda }\end{array}$ & -- & --- & 1 & 7,69\% & 1 & $7,69 \%$ \\
\hline $\begin{array}{l}\text { Hemiplegia desproporcional } \\
\text { incompleta à direita }\end{array}$ & 3 & $23,08 \%$ & -- & --- & 3 & $23,08 \%$ \\
\hline $\begin{array}{l}\text { Hemiplegia desproporcional } \\
\text { incompleta à esquerda }\end{array}$ & 2 & $15,38 \%$ & 1 & $7,69 \%$ & 3 & $23,08 \%$ \\
\hline Total & 9 & $69,23 \%$ & 4 & $30,77 \%$ & 13 & $100 \%$ \\
\hline
\end{tabular}

AVEi: acidente vascular encefálico do tipo isquêmico; AVEh: acidente vascular encefálico do tipo hemorrágico; Fa: frequência absoluta; F\%: frequência relativa.

Ao relacionar tônus muscular aos tipos de AVE, foi 46,16\% (n=6) dos casos de AVEi e em 30,77\% ( $n=4)$ possível verificar que a hipotonia estava presente em dos casos de AVEh (Tabela 3).

Tabela 3: Distribuição da amostra quanto ao tônus muscular em relação ao tipo de AVC.

\begin{tabular}{lccccccc} 
& \multicolumn{2}{c}{ AVEi } & \multicolumn{2}{c}{ AVEh } & \multicolumn{2}{c}{ Total } \\
Tônus muscular & Fa & F\% & Fa & F\% & Fa & F\% \\
\hline Hipotonia & 6 & $46,16 \%$ & 4 & $30,77 \%$ & 10 & $76,93 \%$ \\
\hline Hipertonia & 2 & $15,38 \%$ & --- & -- & 2 & $15,38 \%$ \\
Tônus normal & 1 & $7,69 \%$ & --- & $-\ldots$ & 1 & $7,69 \%$ \\
\hline Total & 9 & $69,23 \%$ & 4 & $30,77 \%$ & 13 & $100 \%$ \\
\hline
\end{tabular}

AVEi: acidente vascular encefálico do tipo isquêmico; AVEh: acidente vascular encefálico do tipo hemorrágico; Fa: frequência absoluta; F\%: frequência relativa. 
Quando analisadas as relaçōes entre os tipos de AVE com a marcha e a sensibilidade, foi observado que se destacaram a marcha helicoidal $(46,15 \% ; n=6)$ e o déficit de sensibilidade proprioceptiva $(38,46 \% ; n=5)$ no AVEi. Já no AVEh, foram mais expressivas a marcha helicoidal $(15,38 \% ; \mathrm{n}=2)$ e a sensibilidade exteroceptiva deficitária (30,77\%; n=4) (Gráfico 2).

Gráfico 2: Distribuição da amostra quanto à sensibilidade e tipo de marcha.

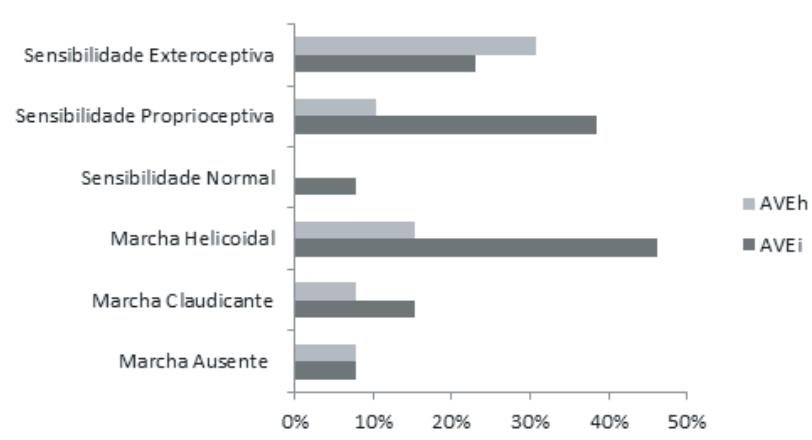

AVEh: acidente vascular encefálico do tipo hemorrágico (coluna cinza-claro); AVEi: acidente vascular encefálico do tipo isquêmico (coluna cinza-escuro).

Com relaçáo às técnicas e recursos fisioterapêuticos utilizadas, foi possível evidenciar que se destacaram a cinesioterapia $(69,23 \% ; n=9)$ e a eletroterapia $(53,85 \% ; n=7)$, seguidos pela mecanoterapia $(46,15 \%$; $=6)$ (Gráfico 3).

Gráfico 3: Distribuição da amostra quanto às técnicas e recursos fisioterápicos utilizados.

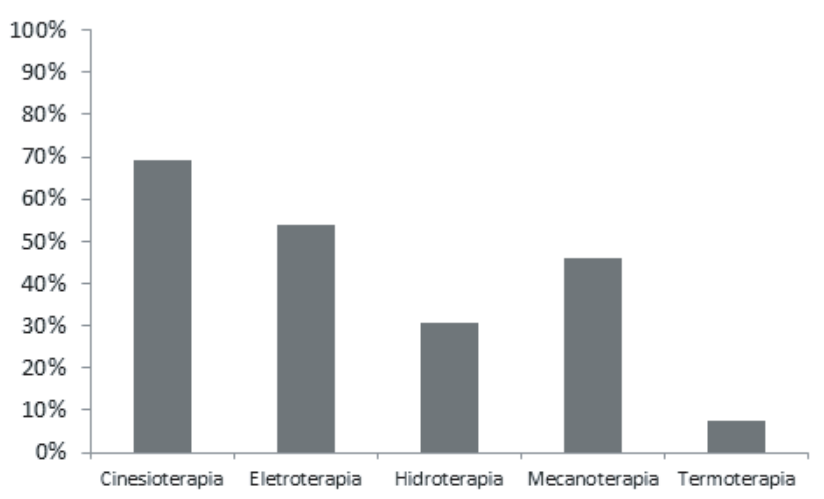

\section{Discussão}

As consequências do AVE podem acarretar sequelas como plegias ou paresias em um ou nos dois membros corporais; alterações espásticas, psicoativas (depressão, ansiedade e agressividade), além das alteraçôes cognitivas, como: déficits de memória, atenção e concentração, disfasia e dificuldades para planejar açóes ${ }^{7}$.
O perfil detectado na amostra apresenta a predominância de homens, e isso pode ser confirmado por outros estudos. É possível associar isso devido a certo distanciamento masculino com a questão do autocuidado, muitas vezes na atenção aos fatores de risco, aos hábitos e estilo de vida e a influência na sua saúde ${ }^{7,8}$.

Entretanto, em relação a faixa etária, os dados diferem da encontrada na literatura - o perfil predominante é de pessoas com idade acima de 60 anos. Embora a idade avançada seja um fator de risco para o acometimento do AVE, o achado deste estudo pode estar mais relacionado ao fator de risco de hipertensão arterial, que vem progressivamente sendo encontrada em indivíduos com mais de 40 anos?.

Foi observado também que a profissão de comerciante acometido por AVCi apresentou a maior prevalência. Falcão et al. ${ }^{10}$ afirmam que há maior prevalência das profissōes do setor de serviços cujas ocupaçōes mais frequentes são de caráter manual, como pedreiro, entre outros, o que implica em uma possível recuperação mais difícil, ao considerar o retorno para o mesmo tipo de atividade laboral.

De acordo com Chaves ${ }^{1}$, a história da doença familiar apresenta grande variabilidade, e poucos estudos epidemiológicos avaliam esse fato. As maiores ocorrências de HAS e AVE foram observadas nos pais de pacientes hipertensos, sugerindo alguns fatores genéticos predisponentes. Esse fato confirma alguns dados evidenciados neste estudo, com maior prevalência da HAS seguida pela DM, tanto no histórico familiar quanto nas comorbidades pré-existentes, dados que podem ser confirmados por Castro, Epstein e Sabino ${ }^{4}$, que afirmam que a HAS é o principal fator de risco modificável para AVE.

Esse fator está também atrelado à maior incidência de casos de AVEi - diagnosticado em 86\% dos casos pesquisados - o que corrobora o achado do estudo, no qual predominou o acometimento de $\mathrm{AVEi}^{11,12}$.

Foi verificado nesta pesquisa que, dentre as sequelas do $\mathrm{AVEi}$, as hemiplegias desproporcionais, incompleta à esquerda e à direita foram as de maior prevalência. As hemiplegias são as principais consequências físicas do AVE e limitam a funcionalidade corporal, dificultando tanto as atividades básicas da vida diária quanto as mais complexas, como as laborais ${ }^{6,13}$.

Referente ao tônus muscular, de acordo com Azevedo ${ }^{14}$, imediatamente após o surgimento da hemiplegia, ocorre a hipotonia (flacidez), cuja duração pode ser de alguns dias ou semanas, evoluindo para a espasticidade (hipertonia) em graus variáveis. De acordo com os dados obtidos nesta pesquisa, verificou-se maior prevalência da hipotonia, tanto no AVEi quanto no AVEh. A hipotonia se apresenta em um primeiro estágio, mais inicial, chamado de "estágio flácido", que gera maior incapacidade 2.

Quanto à força muscular em relaçáo ao tipo de AVC, os membros superiores apresentaram grau dois no AVEh - realização de movimento em amplitude 
completa, sem ação da gravidade -, sendo os mais acometidos, o que vai ao encontro do perfil encontrado em outros estudos, principalmente na regiáo do ombro ${ }^{15}$.

Em relação à marcha, Martins ${ }^{16}$ destaca que a marcha hemiplégica - helicoidal, ceifante - é lenta, abrupta e laboriosa e se caracteriza por uma dificuldade de flexão de quadril, de joelho e tornozelo, associada a uma inclinação anterior e rotação de tronco e abdução exagerada do membro na fase de balanço, o que resulta em assimetria de movimento e diminuição da velocidade. Esse quadro é decorrente dos déficits na percepção-cognição, tônus, controle motor, equilíbrio e mobilidade articular ${ }^{13}$.

Sendo assim, as técnicas e recursos fisioterápicos mais utilizados foram a cinesioterapia, eletroterapia e a mecanoterapia. Benvegnu et al. ${ }^{17}$ apontam que indivíduos que iniciam o tratamento com fisioterapia precocemente e de forma regular, principalmente nos dois primeiros anos após o AVE, apresentam uma melhor evoluçáo na sua independência funcional, considerando que seja comum um menor avanço referente aos cuidados pessoais, mobilidade e locomoção, devido a uma maior complexidade de execução.

Estudos sinalizam a utilização da estimulação elétrica nervosa transcutânea (TENS) no alívio de dor, principalmente no sintoma característico em pacientes com sequelas de AVE, como o ombro doloroso; assim como da estimulação elétrica funcional (FES) para controle muscular, inibição da espasticidade, entre outras ${ }^{18}$. Contudo, ainda não há um consenso da eficácia desses recursos, pois alguns casos apresentam melhora, e outros, não. Marino Junior et al. ${ }^{19}$ apresentaram bons resultados com a cinesioterapia, entretanto a eletroterapia e o uso da tipoia de Bobath não produziram bons resultados no tratamento da síndrome do ombro doloroso em portadores de hemiplegia.

O'Sullivan ${ }^{2}$ afirma que, em práticas baseadas em evidências, é recomendada uma atuação focada em tarefas funcionais específicas, associada a estratégias de aprendizado-motor como base para uma reabilitação mais eficaz. Embora haja um importante papel da fisioterapia nessa doença, é fundamental um acompanhamento multiprofissional.

Acredita-se que os resultados evidenciados por este estudo possam corroborar pesquisas futuras com maior aprofundamento, no sentido de trazer à luz da ciência mais informaçóes sobre os pacientes acometidos por essa doença, lançando mão de diversos instrumentos avaliativos e, assim, poder desenvolver novas metodologias de prevençáo e tratamento, proporcionando melhor qualidade de vida.

\section{Conclusão}

Foi constatado como perfil epidemiológico pacientes do sexo masculino com idade média de 53,77 anos, diagnosticados com AVCi e tendo como profissão o comércio.
Entre as sequelas relacionadas, destacou-se a hemiplegia desproporcional incompleta à esquerda e à direita, a hipotonia, a marcha helicoidal (hemiplégica) e o déficit de sensibilidade proprioceptiva.

\section{Referências}

1. Chaves MLF. Acidente vascular encefálico: conceituaçáo e fatores de risco. Porto Alegre: Rev Bras Hipertens. 2000;4:372-82.

2. O'Sullivan SB. Acidente vascular encefálico. In: O'Sullivan SB, Schmitz TJ. Fisioterapia: avaliação e tratamento. $5^{\mathrm{a}}$ ed. Barueri: Manole; 2010. p. 763-834.

3. Perlini NMOG, Faro ACM. Cuidar de pessoa incapacitada por acidente vascular cerebral no domicílio: o fazer do cuidador familiar. Rev Esc Enferm USP. 2005;39(2):154-63.

4. Castro JAB, Epstein MG, Sabino GB. Estudo dos principais fatores de risco para acidente vascular encefálico. Rev Bras Clin Med. 2009;7(3):171-3.

5. Polese JC, Tonial A, Fung FK, Oliveira SG, Schuster RC. Avaliação da funcionalidade de indivíduos acometidos por Acidente Vascular Encefálico. Rev Neurocienc. 2008;16(3):175-8.

6. Cancela DMG. O Acidente vascular cerebral-classificação, principais consequências e reabilitação. Porto: Psicologia: o portal dos psicólogos [Internet]; 2008 [citado em 2017 mar 1]. Disponível em: http://www.psicologia.pt/artigos/textos/ TL0095.pdf

7. Meneghetti CZM, Delgado GM, Pinto FD, Canonici AP, Gaino MRC. Equilíbrio em indivíduos com acidente vascular encefálico: clínica escola de fisioterapia da Uniararas. Rev Neurocienc. 2009;17(1):14-8.

8. Costa-Junior FM, Maia ACB. Concepçôes de homens hospitalizados sobre a relação entre gênero e saúde. Psic Teo Pesq. 2009;25(1):55-63.

9. Borim FSA, Guariento ME, Almeida EA. Perfil de adultos e idosos hipertensos em unidade básica de saúde. Rev Bras Clin Med. 2011;9(2):107-11.

10. Falcão IV, Carvalho EMF, Barreto KML, Lessa FJD, Leite VMM. Acidente vascular cerebral precoce: implicaçóes para adultos em idade produtiva atendidos pelo Sistema Único de Saúde. Rev Saúde Matern Infant. 2004;4(1):95-102.

11. Gomes A, Nascimento E, Matos L, Martins I, Mós $\mathrm{M}$, Correia J, et al. Acidente vascular cerebral no adulto jovem: estudo prospectivo de 58 doentes. Med Int. 2008;15(3):161-8.

12. Zétola VHF, Nóvak EM, Camargo CHF, Carraro JH, Coral P, Muzzio JA, et al. Acidente vascular cerebral em pacientes jovens: análise de 164 casos. Arq Neuro-Psiquiatr. 2001;59(3B):740-5.

13. Freitas GD. Reabilitação neurofuncional em um paciente com hemiplegia espástica como sequela de AVC: estudo de caso. efdesportes.com. 2011;16(155). 
14. Azevedo RAN. Análise eletromiográfica do movimento de conexão de punho em indivíduos hemiplégicos espásticos [dissertação]. Presidente Prudente: Universidade Estadual Paulista; 2010.

15. Salles FLP, Almeida RL, Ferreira DM. O uso do kineiso tape associado à facilitação neuromuscular proprioceptiva na melhora do controle motor no ombro hemiparético. Rev Bras Reab Ativ Fís. 2012;1(1):42-7.

16. Martins CR, Magnani RM. Análise da marcha de paciente hemiparético decorrente de acidente vascular encefálico: estudo de caso. Universidade Estadual Paulista. Marília; 2008.
17. Benvegnu AB, Gomes LA, Souza CT, Cuadros TBB, Pavão LW, Ávila SN. Avaliação da medida de independência funcional de indivíduos com sequelas de acidente vascular encefálico (AVE). Rev Ciênc Saúde. 2008;1(2):71-7.

18. Schuster RC, Sant CR, Dalbosco V. Efeitos da estimulaçáo elétrica funcional (FES) sobre o padráo de marcha de um paciente hemiparético. Acta Fisiátr. 2007;14(2):82-6.

19. Marino Junior NWM, Ferreira LS, Pastre CM, Valério NI, Lamari NM, Marino LHC. Intervenção fisioterapêutica na síndrome do ombro doloroso em portadores de hemiplegia. Arq Ciênc Saúde. 2005;12(4):220-2.

\section{Como citar este artigo:}

Barbosa RA, Vasconcelos TB, Sousa CT, Costa MFA, Santos MPA, Bastos VPD. Perfil dos pacientes adultos com acidente vascular encefálico tratados em uma clínica-escola de fisioterapia. Rev. Aten. Saúde. 2017;15(51):5-10. 